\title{
Razzi di classe
}

\section{Classy(room) rockets}

\author{
Diego Santimone \\ Scuola media di Losone - Svizzera
}

Sunto / L'esperienza presentata illustra un itinerario didattico, svolto tramite attività cooperative e differenziate, il cui fine era progettare, costruire e lanciare un razzomodello. L'attività è stata incentrata in una cornice narrativa legata allo sviluppo di sé e all'educazione alle scelte tramite la visione del film Cielo d'ottobre (Johnson, 1999). L'esperienza è stata rivolta ad allievi di una terza media (corso base) in maggioranza fortemente demotivata, poco incline all'apprendimento e con progettualità di vita poco sviluppata. La proposta, che si presta ad ampliamenti (inter)disciplinari e trasversali, ha suscitato motivazione e stimolato la cooperazione tra allievi, permettendo così di approfondire diversi ambiti matematici e incentivando la loro crescita personale.

Parole chiave: razzo; progetto didattico; situazione - problema; motivazione.
Abstract / The experience illustrates a didactic itinerary, done through cooperative and differentiated activities, about the project of making and launching a rocket model. The activity was centered in a narrative framework of self-development and education to choices by means of the vision of the film October Sky (Johnson, 1999). The project was designed for students of third-grade middle class (basic math course) strongly demotivated, low inclined to learning and with a lowdeveloped life planning. The proposal, which lends itself to (inter)disciplinary and transversal expansions, has aroused motivation and cooperation of students, making it possible to investigate different mathematical fields and stimulate the students to their personal growth.

Keywords: rocket; project; realistic situations; motivation.

\section{1}

La classe, il contesto e i traguardi di competenza

«Vola solo chi osa farlo» (Sepúlveda, 2010, p. 126). Spesso nei colloqui personali e nei commenti alle verifiche scritte ci si trova a ripensare a questa citazione. Questo è il caso per diversi dei 14 allievi della classe di terza media (corso base) protagonisti di questo articolo, poco o per nulla motivati al conoscere e all'apprendere in matematica, fragili nella collaborazione interpersonale e con bassa capacità di progettualità per il proprio futuro. Dopo qualche mese dall'inizio dell'anno scolastico, si è provato a «farli volare davvero!», pensando a una proposta dove ciascun allievo sarebbe stato chiamato a cooperare con gli altri per realizzare un progetto accattivante. Dopo una fase di ricerca e perlustrazione, si è scelto di proporre un progetto nel quale l'obiettivo fosse riuscire a lanciare in aria un razzomodello; oltre alla riflessione sui traguardi cognitivi del progetto legati al tema, si è ipotizzato di inserire il percorso in un contesto narrativo di sviluppo di sé, di educazione alle scelte e alla cittadinanza. Punto di partenza della riflessione è stato il primo dei traguardi di competenza per la scuola media definiti dal Piano di studio per la scuola dell'obbligo ticinese:

«L'allievo applica il pensiero matematico per comprendere e risolvere con fiducia e determinazione situazioni - problema sia reali sia astratte concernenti 
tutti gli ambiti previsti per il terzo ciclo, mantenendo il controllo critico sia sui processi risolutivi sia sui risultati, esplorando e provando diverse strade risolutive e valutando in modo critico le informazioni e la loro coerenza».

(DECS, 2015, p. 149)

In quest'ottica, il lavoro con gli alunni ha perseguito i seguenti traguardi: I'allievo

- si approccia con interesse all'apprendimento in ambito matematico attraverso il progetto accattivante di un razzomodello;

- mobilita strumenti e competenze matematiche per affrontare problemi situati;

- collabora e coopera positivamente con i suoi compagni;

- è stimolato alla riflessione circa la propria progettualità di vita.

\section{Linee pedagogiche}

La motivazione all'apprendimento è definibile come «il grado di impegno cognitivo investito per il raggiungimento di obiettivi scolastici: spesso ciò viene assunto dagli allievi più come uno stato di lavoro (un dovere) che come un tratto di personalità, un piacere» (Gentile, 1998, p. 81). Ciò rappresenta un nodo cruciale della didattica dal quale «emerge la necessità di comprendere quali scelte didattiche possono creare ambienti di apprendimento motivanti» (Gentile, 1998, p. 83). Un'azione didattica creativa e dotata di molteplici sfumature può prevenire la demotivazione degli studenti ed educare a un interesse ad apprendere. Questa prospettiva di insegnamento si rifà al concetto di coesistenza educativa, ovvero allo sforzo di integrare nell'insegnamento delle discipline scolastiche obiettivi, tecniche e procedure didattiche di natura motivazionale. Ciò si concretizza in alcune attenzioni pedagogiche e scelte didattiche, in particolare:

- convincere gli allievi che sono in grado di affrontare e possono riuscire nei compiti assegnati;

- favorire interazioni positive fra pari e con il docente:

«È attraverso l'incontro, lo scambio, la relazione con gli altri che lo studente impara a valorizzare l'apprendimento in sé stesso e prova gratificazione per l'acquisizione della conoscenza e dello sviluppo delle sue capacità. Tra gli agenti motivanti all'apprendimento i compagni possono essere quelli che influiscono di più».

(Gentile, 1998, p. 87);

- offrire agli allievi una conoscenza e un apprendimento significativi, possibilmente secondo un approccio per scoperta;

- finalizzare il lavoro degli allievi per la realizzazione di qualcosa di condiviso e desiderato.

In questo solco si inserisce la pedagogia del progetto, cioè di una pedagogia che pone al centro l'allievo rendendolo attore e co-costruttore del suo sapere nel perseguire un progetto grazie al lavoro di squadra. In particolare, è il

«compito definito e realizzato in gruppo che implica l'adesione e alla mobilitazione di questi poiché risulta dalla volontà collettiva basata sui desideri giungendo ad un risultato concreto, realizzabile e comunicabile, presentando una utilità rispetto all'esterno del gruppo».

(Licheri 2019, p. 7) 
Affiancato a questo approccio, l'utilizzo di strategie e metodi di apprendimento cooperativo, inserite in situazione, sono un valido strumento per creare modalità di lavoro atte a promuovere la motivazione degli allievi, permettendone l'inclusione e la differenziazione. È così possibile progettare attività didattiche definendo eterogeneamente i ruoli degli allievi all'interno dei singoli gruppi - finalizzando il lavoro ad un compito per il quale ciascun allievo coopera con gli altri secondo competenze distinte - oppure formando, sempre nello stesso percorso didattico, gruppi omogenei a geometria variabile chiamati a collaborare per lo sviluppo di quelle competenze distinte, spendibili poi all'interno del gruppo eterogeneo. II lavoro in piccoli gruppi, le strategie per la gestione del conflitto e di analisi delle modalità di decisione del gruppo sono punti di forza di tale approccio (Comoglio \& Cardoso, 1996). Inoltre, un contesto di apprendimento cooperativo rende possibile la differenziazione didattica, perché permette di strutturare un ambiente didattico nel quale poter accogliere ciascun allievo nella sua unicità e di attivarlo per un apprendimento personalizzato e graduale.

\section{Che cos’è un razzomodello}

Un razzomodello è generalmente un modellino di missile propulso da un motore a razzo chimico in uso presso aeromodellisti (Stine, 2004).

Esistono svariate soluzioni costruttive industriali di razzomodelli, e quella ideale agli obiettivi di questo progetto prevede l'impiego principalmente di cartone e plastica, dimensioni contenute e motori di bassa potenza. II tutto viene prodotto da aziende specializzate a garanzia della sicurezza degli utilizzatori. Si veda la Figura 1.

Figura 1

Razzomodello.

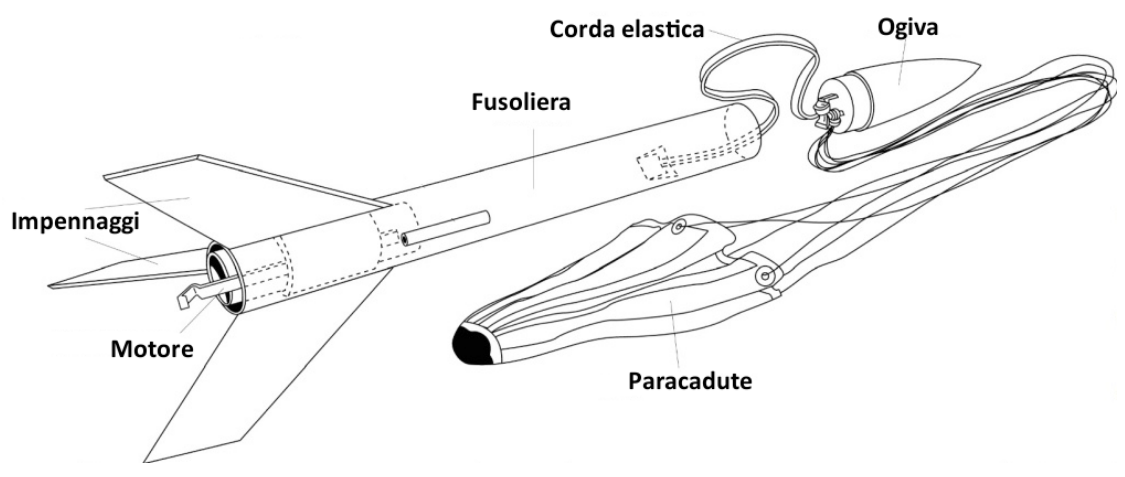

Avviato grazie all'accensione elettrica, il motore a razzo spinge il modello in una certa direzione grazie a una semplice rampa di lancio costituita da una bacchetta metallica. Al termine della generazione della spinta (primissima fase di volo), il motore brucia una carica ritardante durante una fase di volo libero del modello, permettendogli di raggiungere l'apogeo (il punto alla massima distanza verticale dal suolo); un'ultima carica del motore genera del gas all'interno della fusoliera del missile, permettendone il distacco dell'ogiva, il dispiegamento del paracadute in esso contenuto e un atterraggio dolce parzialmente controllato. II generico profilo di missione di un razzomodello è mostrato nella Figura 2 . 
Figura 2

Profilo di missione di un razzomodello (NASA@).
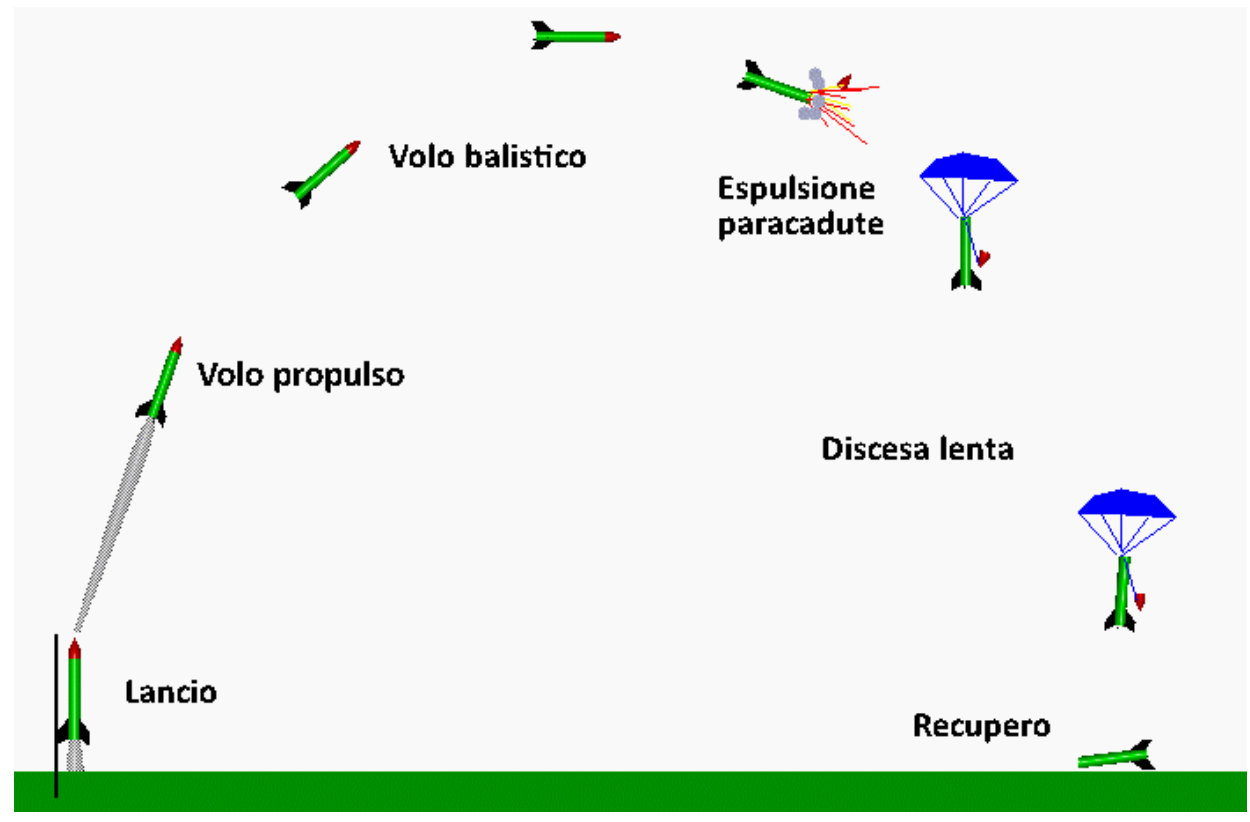

In Figura 3 sono riportate le attrezzature utilizzate per il lancio e la determinazione dell'apogeo. Una particolare pistola-puntatore permette la misura dell'angolo dall'orizzonte, mentre un mini-altimetro con accelerometro triassiale installabile sul razzomodello permette la registrazione della quota di volo e delle accelerazioni nel tempo, con la possibilità di esportare i dati acquisiti in formato elettronico.

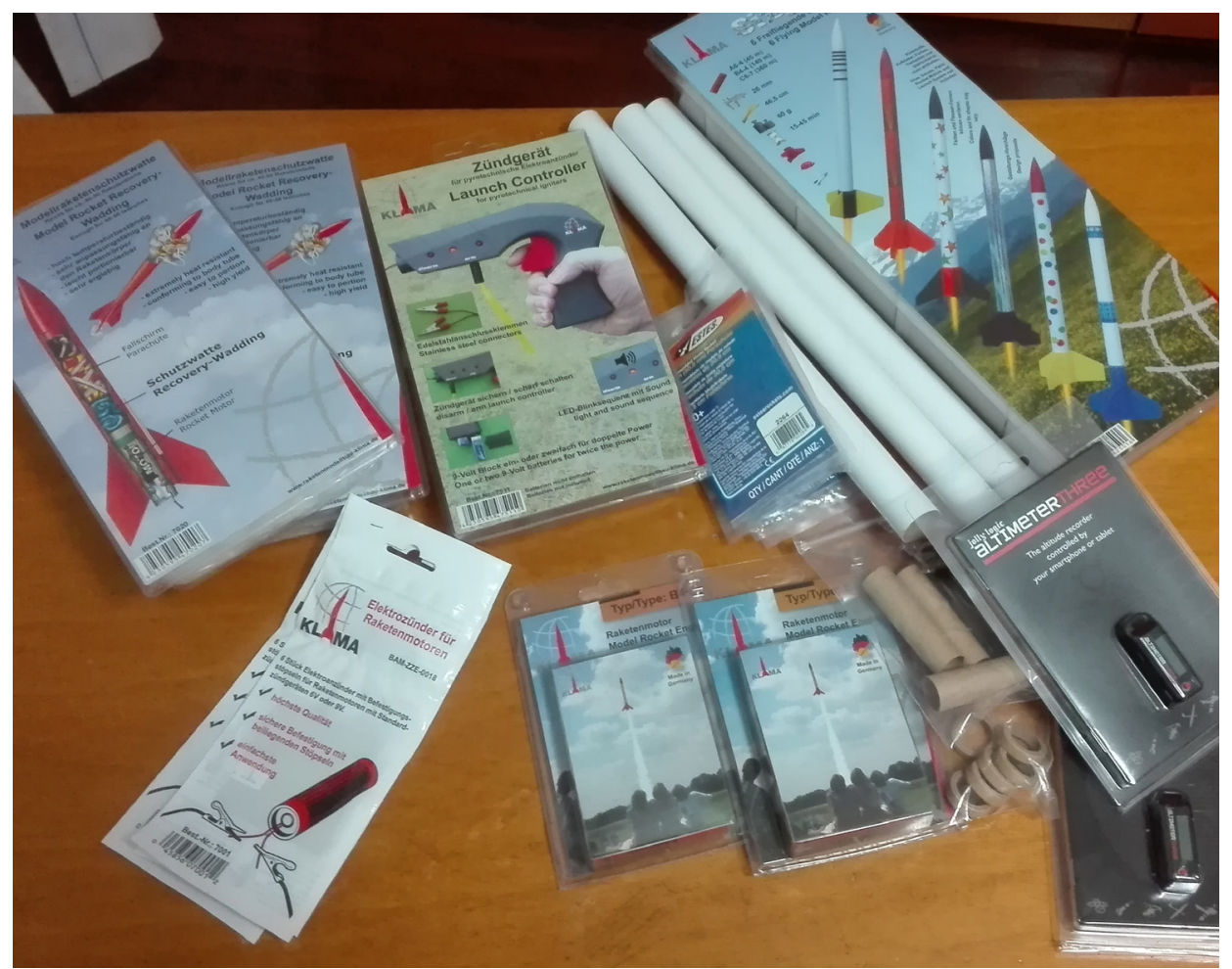

Nella Tabella 1 è riportato il materiale necessario per la realizzazione del progetto. 


\begin{tabular}{|c|c|c|c|}
\hline Item & Costruttore & Modello & Costo (stima) \\
\hline Missili (6 pezzi) & Klima & Six pack Quick \& Easy & $40 \mathrm{CHF}$ \\
\hline Motori (6 pezzi) & Klima & A6-4 & $10 \mathrm{CHF}$ \\
\hline $\begin{array}{l}\text { Rampa e accenditore } \\
\text { elettrico }\end{array}$ & Klima & Mira & $30 \mathrm{CHF}$ \\
\hline Pistola angolare & Estes & $\begin{array}{l}\text { Altitrack Altitude } \\
\text { Finder }\end{array}$ & $25 \mathrm{CHF}$ \\
\hline Altimetro & JollyLogic & AltimeterThree & $100 \mathrm{CHF}$ \\
\hline Materiale vario & - & - & $50 \mathrm{CHF}$ \\
\hline
\end{tabular}

Seppur di massa totale inferiore ai $100 \mathrm{~g}$, le alte velocità in gioco richiedono una cura particolare per l'assemblaggio, l'installazione e il lancio del modello, nonché la verifica preliminare della posizione del suo baricentro ai fini di evitare voli incontrollati o danni a cose e persone. In questo senso, I'educazione alla sicurezza si afferma come un carattere significativo di questo itinerario didattico.

\section{Metodologia}

In linea con il Piano di studio della scuola dell'obbligo ticinese (DECS, 2015), il percorso è stato progettato per essere inserito coerentemente nelle ore di matematica, prevedendo contemporaneamente di non lavorare su obiettivi esclusivi di questa disciplina bensì anche su competenze trasversali e generali; è stato inoltre progettato mantenendo un corretto equilibrio di distribuzione oraria, attraverso la quale si potessero anche preservare le esigenze peculiari della materia. La visione del film Cielo d'ottobre (Johnson, 1999), tratto dall'autobiografia di Hickam (1999), ha fatto da cornice narrativa all'intero percorso. Nel film - ambientato verso la fine degli anni Cinquanta - un gruppo di allievi affascinato dal lancio del primo satellite artificiale Sputnik 1 (4 ottobre 1957) decide di costruire e lanciare dei modelli di razzo, con il sogno di diventare un giorno ingegneri missilistici.

In seguito a questo punto di partenza, l'itinerario si è svolto in tre fasi:

1) Condivisione di senso. Dopo la visione della parte iniziale del film Cielo d'ottobre è stata avanzata la proposta di costruire e lanciare un razzo. Questa fase è stata occasione della formazione dei gruppi, del recupero dei fondi e delle autorizzazioni necessarie.

2) Allenamento e sviluppo. Ogni gruppo ha appreso che cosa fosse un razzomodello e il funzionamento dei suoi sistemi; ha scoperto come determinarne la quota di apogeo tramite semplici misure e costruzioni geometriche; ha verificato il soddisfacimento dei requisiti normativi per il campo di volo e per la sicurezza.

3) Realizzazione. Ogni gruppo ha lanciato il proprio razzo, ne ha determinato la quota di apogeo e ha verificato l'errore percentuale commesso rispetto alla lettura dell'altimetro di bordo. Con la conclusione della proiezione del film e una breve condivisione gli allievi sono stati infine stimolati alla cura della propria progettualità di vita. 
La dimensione riflessiva circa il lavoro e i risultati ottenuti ha permeato ogni fase dell'itinerario.

\section{Descrizione dell'itinerario}

\subsection{Percorso didattico}

Il percorso didattico è composto da attività molto eterogenee fra di loro per quanto riguarda tipologia e tempistiche, ma tutte essenziali per la realizzazione progressiva del progetto di lanciare un razzo da parte di ciascun team di lavoro. Questa eterogeneità ha permesso di inserire agilmente le attività all'interno della calendarizzazione delle lezioni, istituendo momenti più centrati sul progetto ed altri meno, anche in funzione della motivazione della classe riscontrata nelle altre ore di lezione.

\subsection{Inquadramento nel Piano di studio della scuola dell'obbligo ticinese}

Come già anticipato, l'intero progetto intende favorire il primo dei traguardi di competenza per la scuola media definiti dal Piano di studio per la scuola dell'obbligo ticinese (DECS, 2015) legato alla risoluzione dei problemi.

In aggiunta al chiarimento sul traguardo di competenza mobilitato, nella Tabella 2 è riportato sinteticamente l'inquadramento delle attività del progetto nel Piano con particolare attenzione ai nuclei tematici toccati.

Tabella 2

Inquadramento nel Piano di studio della scuola

dell'obbligo ticinese

(DECS, 2015) delle attività

secondo gli ambiti di

competenza mobilitati.

\begin{tabular}{|c|c|c|c|c|c|}
\hline \multirow[t]{2}{*}{ Attività } & & \multicolumn{4}{|c|}{ Ambiti disciplinari coinvolti } \\
\hline & & $\begin{array}{l}\text { Numeri } \\
\text { e calcolo }\end{array}$ & Geometria & $\begin{array}{l}\text { Grandezze } \\
\text { e misure }\end{array}$ & Funzioni \\
\hline \multirow{2}{*}{$\begin{array}{l}\text { Condivisione } \\
\text { di senso }\end{array}$} & $\begin{array}{l}\text { Recupero } \\
\text { fondi }\end{array}$ & & & & \\
\hline & $\begin{array}{l}\text { Richiesta auto- } \\
\text { rizzazione }\end{array}$ & & & & \\
\hline \multirow{5}{*}{$\begin{array}{l}\text { Allenamento } \\
\text { e sviluppo }\end{array}$} & $\begin{array}{l}\text { Scoperta del } \\
\text { razzo }\end{array}$ & & & & \\
\hline & $\begin{array}{l}\text { Determinare } \\
\text { I'apogeo }\end{array}$ & & & & \\
\hline & $\begin{array}{l}\text { Baricentro e } \\
\text { considerazioni } \\
\text { geometriche }\end{array}$ & & & & \\
\hline & $\begin{array}{l}\text { Requisiti del } \\
\text { campo di volo }\end{array}$ & & & & \\
\hline & $\begin{array}{l}\text { Definizione } \\
\text { delle operazio- } \\
\text { ni di lancio }\end{array}$ & & & & \\
\hline \multirow{2}{*}{ Realizzazione } & $\begin{array}{l}\text { Installazione e } \\
\text { lancio }\end{array}$ & & & & \\
\hline & $\begin{array}{l}\text { Analisi } \\
\text { post-lancio }\end{array}$ & & & & \\
\hline
\end{tabular}


Infine, nella Tabella 3 si riportano le competenze trasversali attivate e i contesti di formazione generale coinvolti durante le attività.

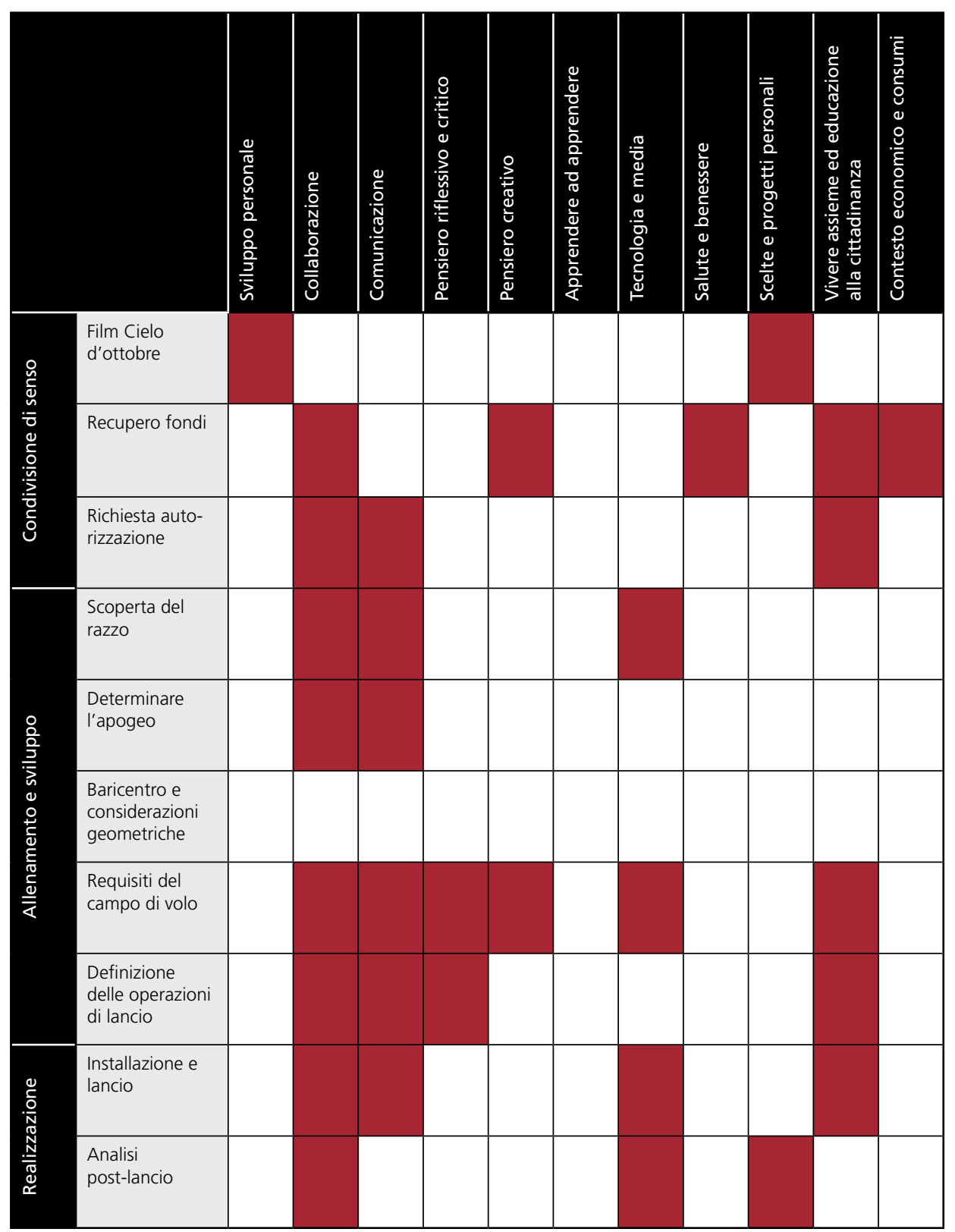

Inquadramento nel Piano

di studio della scuola

dell'obbligo ticinese

(DECS, 2015) delle

attività: competenze

trasversali e contesti di

Nella presentazione delle singole attività si descriveranno in maggior dettaglio le risorse ed i processi cognitivi attivati di volta in volta. Per ciascuna verrà definito l'obiettivo finalizzato al progetto del lancio del razzo, le tempistiche ed il materiale utilizzato, la descrizione dell'attività stessa e il suo inquadramento nel Piano di studio della scuola dell'obbligo ticinese.

\subsection{Condivisione di senso: recupero dei fondi}

Obiettivo progettuale

Recuperare i fondi economici necessari all'acquisto del materiale. 


\section{Tempistiche e materiale}

Mezza ora-lezione in classe per decidere insieme la strategia da adottare; un'ora-lezione di considerazioni matematiche; una/mezza giornata extra-scolastica per il banco del dolce.

II materiale per il lavoro in classe consiste in schede didattiche (Allegati 1 e 2); I'occorrente per il banco del dolce viene recuperato direttamente dagli allievi (dolci, fazzoletti, piatti ecc.).

\section{Descrizione dell'attività}

Dopo la visione della prima parte del film Cielo d'ottobre (circa 15 minuti), è stato proposto agli allievi di provare a costruire e lanciare un razzo. Con l'entusiasmo di tutti ha preso avvio l'itinerario. La classe è stata subito attivata alla responsabilità del progetto con una domanda: "Ci saranno delle spese da sostenere: come recuperiamo i fondi per il nostro progetto?». Tutte le proposte avanzate liberamente dagli allievi sono state confrontate in termini di costi-benefici fino alla scelta finale condivisa di organizzare un banco del dolce presso un centro commerciale del comune di Losone in una giornata di sabato. Gli allievi si sono suddivisi compiti e materiali, redigendo una tabella di turni di servizio.

In vista dell'evento è stata proposta alla classe un'attività di matematica inerente a situazioni di riduzione all'unità di grandezze direttamente proporzionali allo scopo di preparare le torte per diverse persone a partire da una ricetta per quattro persone. Suddivisi a coppie omogenee, gli allievi hanno dovuto affrontare situazioni concernenti tre tipologie di dolce rivolte a sviluppare diversi traguardi di apprendimento:

- Torta di mandorle. Individuare strategie per la risoluzione di un problema (quantificare gli ingredienti necessari per un certo numero di persone) e sperimentarne la validità con un crescendo di complessità di richieste.

- Torta di carote. Generalizzare il procedimento scoperto con la prima torta. Calcolo degli elementi nutrizionali presenti in ciascuna fetta a partire dalle informazioni riportate sulle confezioni degli ingredienti.

- Ciambellotto. Riflettere sui rapporti fra ingredienti usati per differenti dosi. Scoprire un metodo grafico (diagramma cartesiano) per la determinazione delle quantità di ingredienti (grandezze direttamente proporzionali).

I ragazzi, divisi a coppie, hanno affrontato in sequenza le tre ricette e le situazioni ad esse riferite, favorendo così una differenziazione progressiva e permettendo a ciascuna coppia da un lato di completare velocemente i lavori che presentavano attività matematiche con cui avevano già confidenza, dall'altro di soffermarsi in quelle che richiedevano tentativi, scoperte e nuovi apprendimenti.

Per maggiori dettagli si veda il piano lezione nell'Allegato 1 e le schede didattiche nell'Allegato 2.

Il banco del dolce è stato anche occasione per pubblicizzare il progetto verso i compagni e i familiari degli allievi, come si vede in Figura 4 nella pagina seguente. 
Figura 4

Banco del dolce e

promozione del progetto.

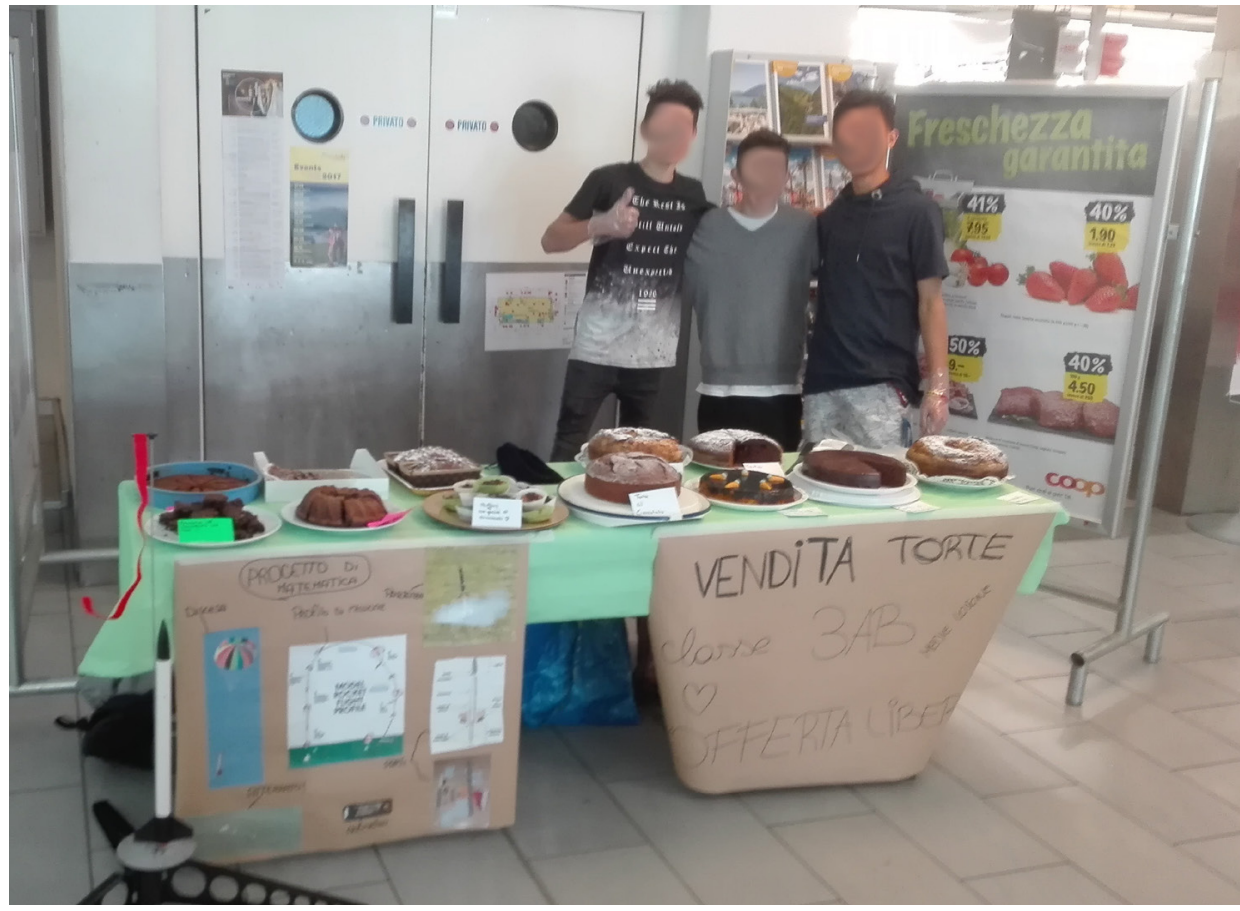

Inquadramento nel Piano di studio della scuola dell'obbligo ticinese Come mostrato nella Tabella 4, questa attività si inserisce negli ambiti Numeri e cal- colo, Grandezze e misure e Funzioni.

\begin{tabular}{l|l|l|l}
\hline $\begin{array}{l}\text { MODELLO DI } \\
\text { COMPETENZA }\end{array}$ & Numeri e calcolo & Grandezze e misure & Funzioni \\
\hline Sapere e riconoscere & $\begin{array}{l}\text { Riconoscere frazioni } \\
\text { equivalenti }\end{array}$ & & \\
\hline Eseguire e applicare & $\begin{array}{l}\text { Eseguire semplici calcoli } \\
\text { aritmetici }\end{array}$ & $\begin{array}{l}\text { Convertire unità di } \\
\text { misura }\end{array}$ & \\
\hline Esplorare e provare & $\begin{array}{l}\text { Individuare strategie } \\
\text { per la risoluzione di un } \\
\text { problema di riduzione } \\
\text { all'unità }\end{array}$ & & \\
\hline $\begin{array}{l}\text { Matematizzare } \\
\text { e modellizzare }\end{array}$ & $\begin{array}{l}\text { Generalizzare un } \\
\text { procedimento tramite } \\
\text { una formula algebrica } \\
\text { applicabile a situazioni } \\
\text { simili }\end{array}$ & & $\begin{array}{l}\text { Elaborare un metodo } \\
\text { grafico (diagramma } \\
\text { cartesiano) per la deter- } \\
\text { minazione di grandezze } \\
\text { proporzionali }\end{array}$ \\
\hline $\begin{array}{l}\text { Interpretare e } \\
\text { riflettere sui risultati }\end{array}$ & $\begin{array}{l}\text { Descrivere con linguag- } \\
\text { gio naturale il procedi- } \\
\text { mento adottato }\end{array}$ & & \\
\hline $\begin{array}{l}\text { Comunicare } \\
\text { e argomentare }\end{array}$ & - Collaborazione \\
\hline $\begin{array}{l}\text { Competenze } \\
\text { trasversali }\end{array}$ & - Pensiero creativo & & \\
\hline $\begin{array}{l}\text { Contesti di } \\
\text { formazione generali }\end{array}$ & - Vivere assieme ed educazione alla cittadinanza & \\
\hline Contesto economico e consumi & & \\
\hline
\end{tabular}

Tabella 4

Recupero dei fondi: inquadramento nel Piano di studio della scuola dell'obbligo ticinese. 


\subsection{Condivisione di senso: richiesta autorizzazione}

Obiettivo progettuale

Verificare la liceità ad operare con razzomodelli sul sedime scolastico.

\section{Tempistiche e materiale}

Mezza ora-lezione.

\section{Descrizione dell'attività}

Partendo dalla provocazione del docente «Ma possiamo lanciare liberamente un razzomodello oppure occorre rispettare alcune norme?» la classe ha recepito, dopo qualche resistenza, la necessità di rivolgersi a qualcuno per chiedere la possibilità di procedere al progetto. Dopo un breve confronto circa chi interrogare, la scelta è caduta sull'autorità locale: i ragazzi, divisi in gruppi, hanno scritto una proposta di lettera di richiesta di autorizzazione e ciascun contributo è stato poi utilizzato nelle sue parti più riuscite per la stesura della lettera finale. D'accordo con il docente, l'autorità locale ha inoltrato la risposta alla domanda della classe dando il via libera a patto di rispettare il codice di regolamentazione internazionale NAR (National Association of Rocketry, Allegato 3) per razzimodellismo, evidenziando che la responsabilità di tutto sarebbe stata del docente.

Inquadramento nel Piano di studio della scuola dell'obbligo ticinese Questa attività non attiva competenze specificatamente matematiche ma trasversali, operando in contesti di formazione generali, come mostrato nella Tabella 5.

\section{Tabella 5}

Richiesta dell'autorizza-

zione: inquadramento nel

Piano di studio della

scuola dell'obbligo

ticinese.

\begin{tabular}{|l|l|}
$\begin{array}{l}\text { Competenze } \\
\text { trasversali }\end{array}$ & - Comunicazione \\
& - Collaborazione \\
\hline $\begin{array}{l}\text { Contesti di } \\
\text { formazione generali }\end{array}$ & - Vivere assieme ed educazione alla cittadinanza \\
\hline
\end{tabular}

\subsection{Allenamento e sviluppo: scoperta e costruzione del razzo}

Obiettivo progettuale

Scoprire le parti e il funzionamento del razzomodello; creare i gruppi di lavoro; assemblare i razzomodelli per il lancio.

\section{Tempistiche e materiale}

Un'ora-lezione per la scoperta del razzomodello; un'ora-lezione per il suo assemblaggio.

Un kit di assemblaggio di un razzomodello per ogni gruppo; schede didattiche.

\section{Descrizione dell'attività}

Una presentazione da parte del docente ha avviato la prima ora-lezione facendo intuire agli allievi lo sfruttamento da parte di un motore a razzo del terzo principio della dinamica. II profilo di missione (si veda Figura 2) è stato presentato per introdurre I'analisi delle parti principali e del funzionamento del razzomodello. Gli allievi sono stati inizialmente suddivisi in coppie e a ciascuna di esse è stata affidata una parte fisica del modello e una descrizione funzionale della stessa. Dopo una decina di minuti di analisi del materiale, ciascuna coppia è intervenuta per pochi minuti spiegando al 
resto della classe la parte che aveva studiato mentre alla lavagna veniva disegnato dal docente lo schema di un razzomodello via via che le presentazioni si succedevano. Nella seconda ora-lezione il docente ha presentato la composizione dei gruppi di lancio - da lui predefinita per garantirne l'eterogeneità - che hanno proceduto ad assemblare il proprio razzomodello a partire dal kit e dalle istruzioni fornite dal costruttore (si veda Figura 5). Nel tempo rimanente i gruppi hanno scelto il nome del proprio razzo e progettato una patch (logo, distintivo) di missione che hanno poi preparato a casa.

Le schede didattiche utilizzate in queste ore-lezione sono riportate nell'Allegato 4.

Figura 5

Allenamento e sviluppo: scoperta e costruzione del razzomodello, attività in aula.

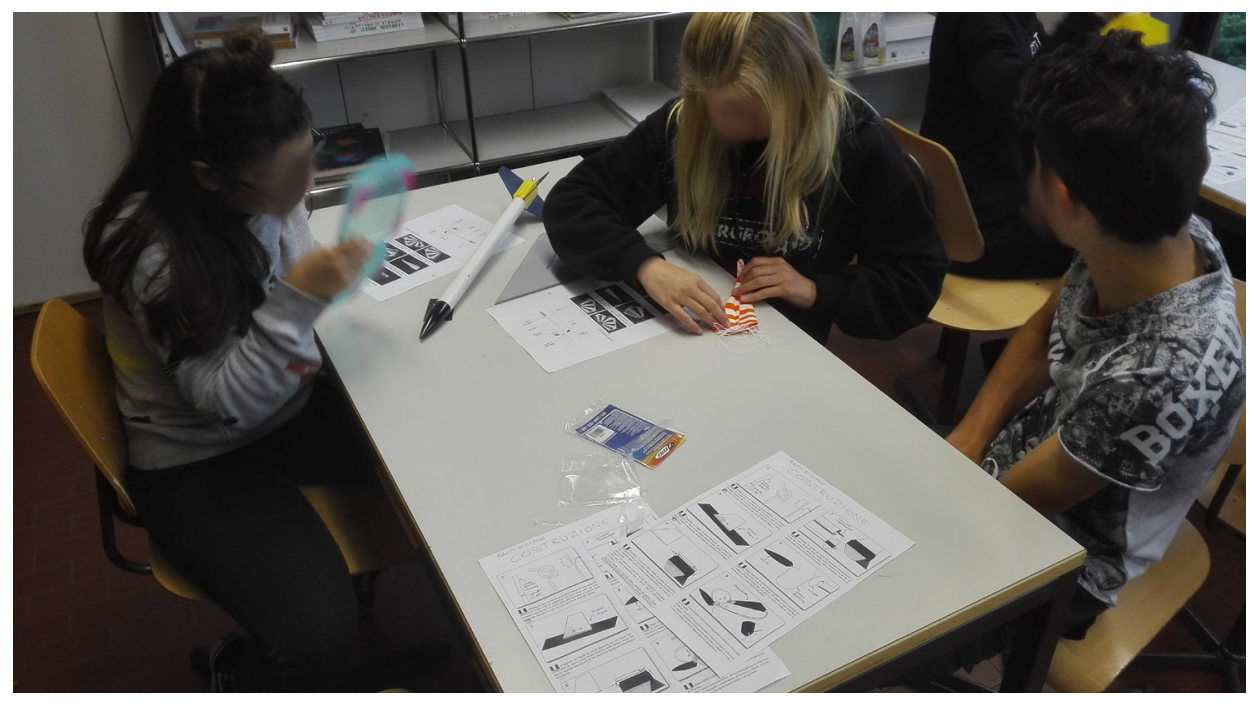

Inquadramento nel Piano di studio della scuola dell'obbligo ticinese Questa attività non attiva competenze specificatamente matematiche ma trasversali, come evidenziato nella Tabella 6 .

\begin{tabular}{|l|l|}
$\begin{array}{l}\text { Competenze } \\
\text { trasversali }\end{array}$ & $\begin{array}{l}- \text { Comunicazione } \\
- \text { Collaborazione }\end{array}$ \\
\hline $\begin{array}{l}\text { Contesti di } \\
\text { formazione generali }\end{array}$ & - Tecnologia e media \\
\hline
\end{tabular}

\subsection{Allenamento e sviluppo: determinazione dell'apogeo}

Obiettivo progettuale

Definire una procedura matematica per la misurazione della quota di apogeo raggiunta da un razzomodello.

\section{Tempistiche e materiale}

Due ore-lezione. Schede didattiche; goniometri; carta millimetrata; righe millimetrate; pistola per la misurazione angolare.

\section{Descrizione dell'attività}

Insieme agli allievi è stata problematizzata la questione di poter calcolare l'apogeo, 
cioè la massima quota raggiunta dal razzomodello durante il volo. Per la scoperta di una procedura matematica affidabile è stato scelto un approccio di apprendimento cooperativo secondo la struttura jigsaw' ${ }^{1}$. La classe è stata suddivisa in tre gruppi omogenei, chiamati ad esplorare diversi strumenti e risorse matematiche:

- Un gruppo - gli allievi con più attitudine per la matematica - ha esplorato la pistola per la misurazione angolare (Figura 6), strumento che permette di determinare l'ampiezza angolare di un certo punto nel cielo rispetto all'orizzonte. Puntandola verso il razzomodello durante la sua ascesa è possibile misurare l'angolo definito dall'orizzonte e dal modello con l'osservatore nel suo vertice. Con uno schema geometrico e una pistola come artefatto gli allievi di questo gruppo dovevano capire quale angolo venisse misurato dalla pistola, così da esser sicuri del suo corretto utilizzo.

Figura 6

Pistola per la misurazione angolare.

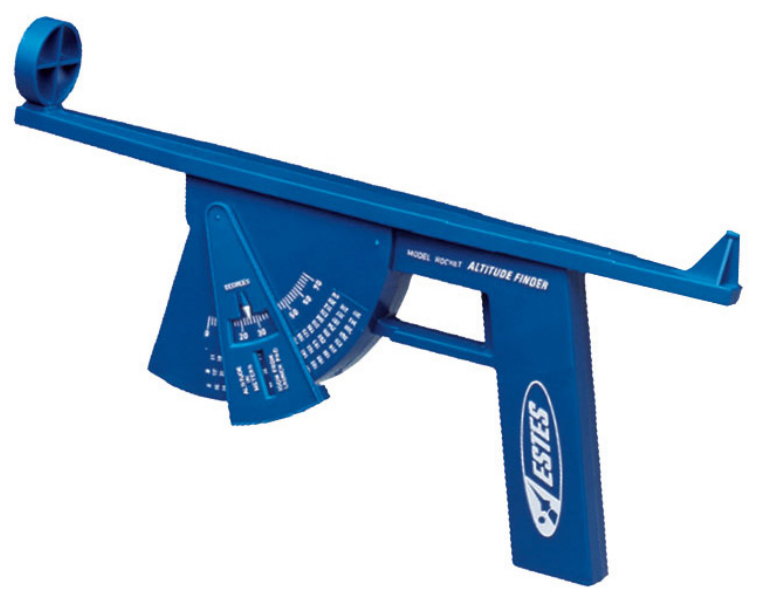

- Un gruppo di competenze intermedie ha lavorato all'utilizzo della carta millimetrata e del diagramma cartesiano come strumenti per conoscere le coordinate e quindi la posizione di un punto nello spazio a partire da certe informazioni (rapporti di scala, spostamenti e pendenze).

- Il gruppo degli allievi con più difficoltà in matematica ha esplorato alcuni triangoli generici (ri)scoprendo che la somma delle ampiezze degli angoli interni è pari a $180^{\circ}$.

Dopo una prima fase di lavoro nei gruppi omogenei gli allievi sono stati riassegnati in terne eterogenee composte da allievi derivanti da ciascuno dei gruppi iniziali. Ai nuovi gruppi è stato posto l'obiettivo di scoprire un modo per determinare l'altitudine massima raggiunta dal razzomodello, disponendo solamente di due pistole di misura angolare, la posizione della rampa di lancio e di due punti di osservazione, riga e carta millimetrata. I gruppi hanno lavorato con interesse, presumibilmente attivati e motivati dalla situazione sfidante e dalle conoscenze/abilità che ciascun allievo aveva acquisito o consolidato nell'attività precedente: ciascuno di essi poteva fornire un contributo unico e insostituibile al gruppo di lavoro. Dopo una fase di esplorazione e

1. Il metodo del jigsaw in ottica di cooperative learning prevede che gli allievi si suddividano in gruppi omogenei di lavoro, all'interno dei quali sviluppino particolari competenze (distinte fra gruppi) spendibili in un secondo momento in altri gruppi eterogenei (composti da allievi derivati da ciascuno dei gruppi omogenei) chiamati ad affrontare una situazione complessa nella quale ciascun allievo contribuisce con I'apprendimento e le competenze ottenute nel gruppo precedente. 
scoperta, tutti i gruppi (ad eccezione di uno) sono giunti alla definizione del metodo di determinazione dell'apogeo (Figura 7) che è stato condiviso e raffinato con una messa in comune finale:

- In due postazioni ( $\alpha$ e $\beta$ ) - la cui distanza lineare dalla rampa di lancio è nota due allievi misurano l'ampiezza angolare determinata dall'apogeo del razzomodello grazie a due pistole.

- Sulla carta millimetrata viene rappresentata in scala la distanza fra le due postazioni di misura e vengono riportati gli angoli le cui ampiezze sono state misurate nelle postazioni $\alpha$ e $\beta$ rispetto al suolo.

- Prolungando e intersecando i lati dei due angoli si determina il punto di apogeo del razzomodello nel cielo, la cui altezza rispetto al suolo può essere misurata sulla carta millimetrata e rapportata in scala alla realtà.

Infine si è riflettuto sull'inevitabilità di commettere errori di misura o legati alla rappresentazione in questa procedura. Per migliorare la precisione della misurazione sul campo si è deciso inoltre di inclinare di qualche grado rispetto alla verticale la rampa di lancio, in modo tale da garantire con un certo grado di fiducia che la traiettoria di ascesa del razzo fosse interamente contenuta nel piano definito dai due angoli.

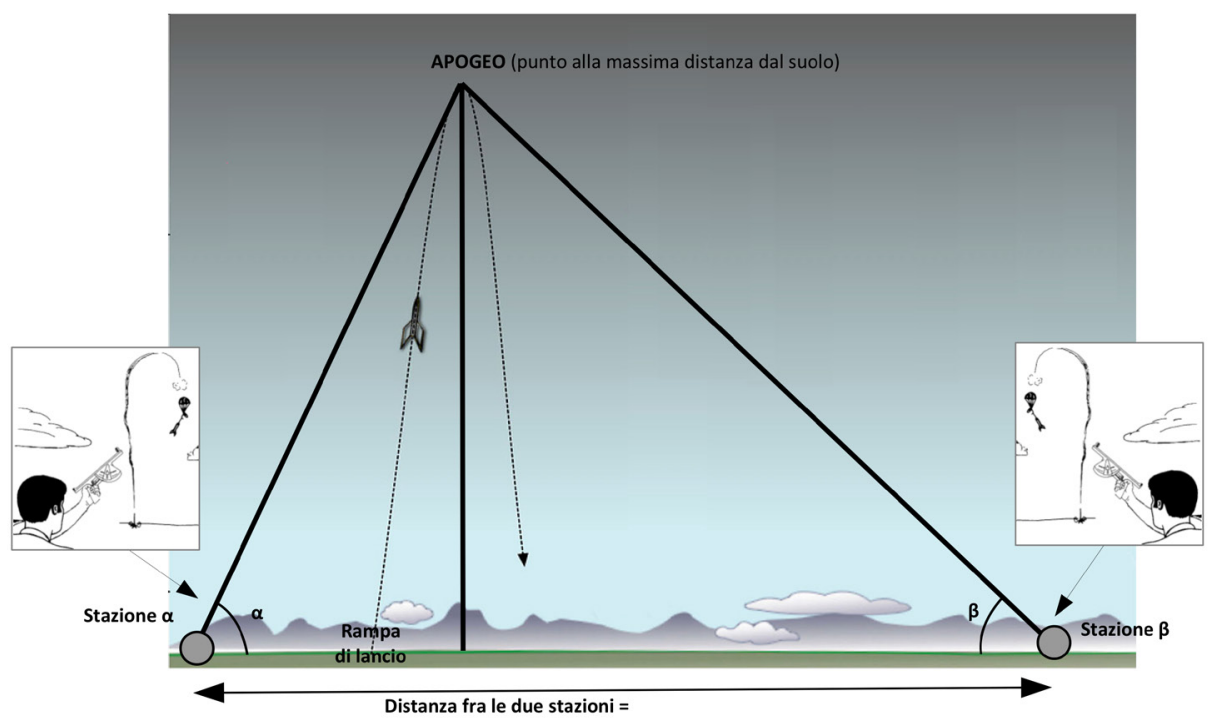

Le schede didattiche utilizzate in queste ore-lezione sono riportate nell'Allegato 5.

Inquadramento nel Piano di studio della scuola dell'obbligo ticinese Come si può facilmente evincere dalla descrizione precedente, e come mostrato in Tabella 7, questa attività si inserisce negli ambiti Geometria e Grandezze e misure. 
Tabella 7

Allenamento e sviluppo: determinare I'apogeo.

Inquadramento nel Piano

di studio della scuola

dell'obbligo.
Figura 8 (a sinistra) Stabilità di un

razzomodello in funzione della posizione del baricentro (CG) e del centro di pressione $(\mathrm{CP})$. Immagini tratte da Barrowman (1970)

\section{Figura 9 (a destra)} Determinazione della posizione del baricentro (CG) e del centro di pressione $(C P)$ in un razzomodello. Immagini tratte da Barrowman (1970).

\begin{tabular}{|c|c|c|}
\hline $\begin{array}{l}\text { MODELLO DI } \\
\text { COMPETENZA }\end{array}$ & Numeri e calcolo & Grandezze e misure \\
\hline Sapere e riconoscere & & $\begin{array}{l}\text { Comprendere che la somma delle ampiezze degli } \\
\text { angoli interni di un triangolo è pari a } 180^{\circ}\end{array}$ \\
\hline Eseguire e applicare & $\begin{array}{l}\text { Individuare rapporti di } \\
\text { scala, pendenze }\end{array}$ & $\begin{array}{l}\text { Rappresentare in scala una certa situazione. Saper } \\
\text { leggere e disegnare un diagramma cartesiano }\end{array}$ \\
\hline Esplorare e provare & & Scoprire il funzionamento della pistola angolare \\
\hline $\begin{array}{l}\text { Matematizzare } \\
\text { e modellizzare }\end{array}$ & & $\begin{array}{l}\text { Modellizzare la situazione del volo del razzomodello } \\
\text { definendone gli elementi e i dati essenziali }\end{array}$ \\
\hline $\begin{array}{l}\text { Interpretare e } \\
\text { riflettere sui risultati }\end{array}$ & & $\begin{array}{l}\text { Avere consapevolezza delle tipologie di errori intro- } \\
\text { dotti nella procedura }\end{array}$ \\
\hline $\begin{array}{l}\text { Comunicare } \\
\text { e argomentare }\end{array}$ & & $\begin{array}{l}\text { Condividere le conoscenze/competenze con gli altri } \\
\text { compagni }\end{array}$ \\
\hline $\begin{array}{l}\text { Competenze } \\
\text { trasversali }\end{array}$ & $\begin{array}{l}\text { - Comunicazione } \\
\text { - Collaborazione }\end{array}$ & \\
\hline
\end{tabular}

\subsection{Allenamento e sviluppo: baricentro e considerazioni geometriche} Obiettivo progettuale

La stabilità di volo di un razzomodello deve essere verificata prima del lancio così da garantire che - in assenza di vento - il modello mantenga la direzione stabilita dalla rampa di lancio, senza percorrere traiettorie casuali e potenzialmente pericolose per cose e persone. Per far ciò si utilizza nel modellismo una procedura molto semplice che richiede la determinazione della posizione assiale del baricentro (CG) e del centro di pressione (CP) del razzomodello: affinché il modello sia stabile occorre che il CP sia arretrato al CG rispetto all'ogiva (Figura 8). II CG viene determinato sperimentalmente ricercando l'equilibrio del razzomodello completo appeso ad un filo, mentre la posizione assiale del CP viene ricercata come il baricentro della proiezione laterale (bidimensionale) del modello (Figura 9). Per maggiori informazioni si vedano i lavori di Barrowman (1970); Grimm (1999); Nolte (2012).
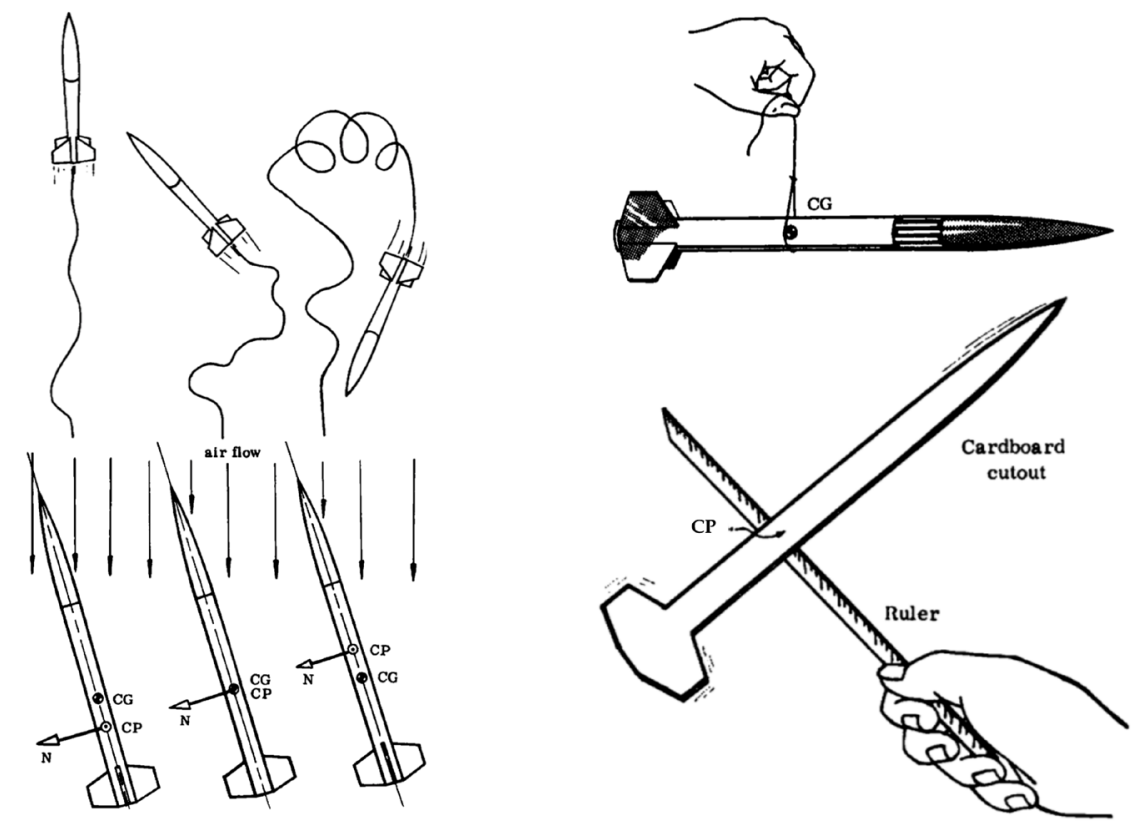


\section{Tempistiche e materiale}

Due ore-lezione. Schede didattiche; razzomodelli; spago; cartoncino (3-4 mm).

\section{Descrizione dell'attività}

Tramite una lezione dialogata gli allievi sono stati introdotti alla determinazione del baricentro di un corpo eterogeneo a partire dal riconoscimento di simmetrie bi/tridimensionali e alla determinazione qualitativa dei centri di simmetria di diverse tipologie di corpi. II concetto di equilibrio stabile, instabile e indifferente è stato poi proposto a partire dall'esperienza dell'attacco di un quadro ad una parete. Consolidato il concetto di baricentro, i ragazzi - nuovamente divisi in gruppi - hanno poi proceduto alla determinazione sperimentale del baricentro (CG) e del centro di pressione $(\mathrm{CP})$ del proprio razzomodello. Si vedano le schede didattiche riportate negli Allegati 6 e 7.

Inquadramento nel Piano di studio della scuola dell'obbligo ticinese Anche questa attività si inserisce nell'ambito Geometria, come riportato nella tabella sottostante.

\begin{tabular}{|l|l|}
\hline $\begin{array}{l}\text { MODELLO DI } \\
\text { COMPETENZA }\end{array}$ & Geometria \\
\hline Sapere e riconoscere & Individuare gli assi e i centri di simmetria di figure bidimensionali \\
\hline Eseguire e applicare & $\begin{array}{l}\text { Ipotizzare la posizione del baricentro di un corpo a partire dalle sue } \\
\text { caratteristiche geometriche e dei materiali } \\
\text { Ricercare sperimentalmente il baricentro di una figura bidimensionale } \\
\text { e di un corpo non omogeneo }\end{array}$ \\
\hline Esplorare e provare & $\begin{array}{l}\text { Matematizzare } \\
\text { e modellizzare }\end{array}$ \\
\hline $\begin{array}{l}\text { Interpretare e } \\
\text { riflettere sui risultati }\end{array}$ & \\
\hline $\begin{array}{l}\text { Comunicare e } \\
\text { argomentare }\end{array}$ & \\
\hline
\end{tabular}

\subsection{Allenamento e sviluppo: requisiti del campo di volo}

Obiettivo progettuale

Verificare il soddisfacimento dei requisiti del campo di volo previsti dal regolamento NAR.

\section{Tempistiche e materiale}

Mezz'ora-lezione. Foto aree della sede scolastica.

\section{Descrizione dell'attività}

Alla classe è stato chiesto di leggere il codice di regolamentazione NAR (Allegato 3) scoprendo così che il campo di volo avrebbe dovuto rispettare alcuni requisiti in termini di distanze, aree e condizioni meteorologiche. L'intenzione era di effettuare i lanci nel parco della sede scolastica, ma sarebbe stato prima necessario verificare il 
soddisfacimento dei requisiti del regolamento date le caratteristiche dell'area e del posizionamento della rampa di lancio.

Il docente ha suddiviso in coppie eterogenee gli allievi e a ciascuna di esse ha fornito una ripresa aerea della sede scolastica, nella quale però era stata nascosta l'indicazione del rapporto di scala (si veda la Figura 10). A partire dalla sola fotografia, gli allievi dovevano proporre la stima delle dimensioni del parco e stabilire in quale posizione andasse collocata la rampa di lancio in accordo con i requisiti NAR. Per svolgere questa attività di stima le coppie hanno capito che dovevano calcolare il rapporto di scala della fotografia utilizzando delle unità di misura di lunghezza inusuali dal punto di vista della misurazione scientifica: la lunghezza di un'automobile, la pista dei 100 metri, una panchina del piazzale ecc. Le strategie adottate e i risultati ottenuti sono stati poi condivisi in una messa in comune nella quale è stato possibile riflettere sulla bontà dei risultati ottenuti a seconda delle diverse lunghezze note considerate e della deformazione prospettica dell'immagine.

Figura 10

Ripresa area della sede scolastica

(GoogleEarth@)
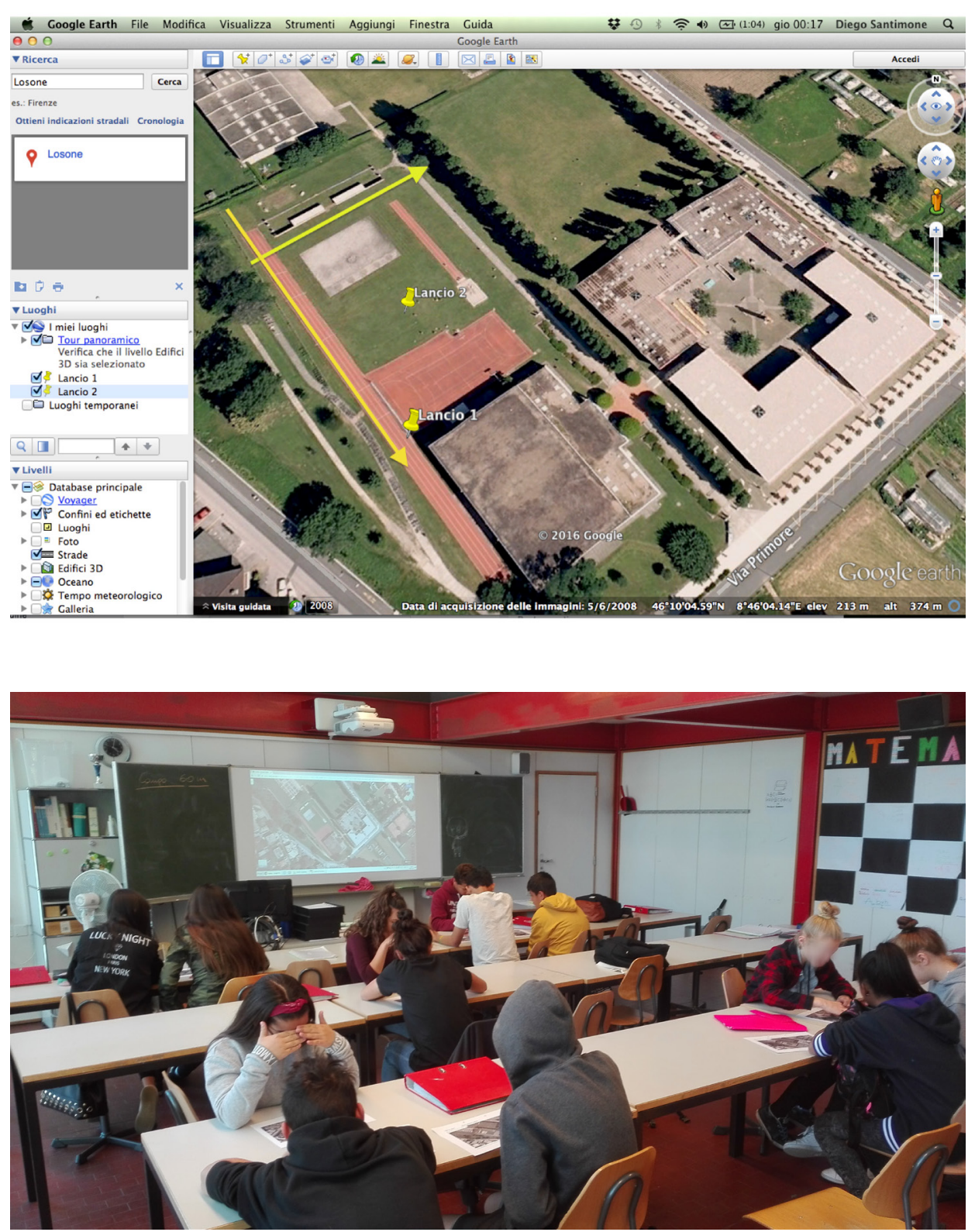

\section{Figura 11}

Verifica dei requisiti NAR per il campo di volo. 
Inquadramento nel Piano di studio della scuola dell'obbligo ticinese Questa attività si inserisce negli ambiti Geometria e Grandezze e misure (Tabella 9).

Tabella 9

Allenamento e sviluppo: requisiti del campo di

volo. Inquadramento nel

Piano di studio della scuola dell'obbligo

ticinese.

\begin{tabular}{l|l|l|}
\hline $\begin{array}{l}\text { MODELLO DI } \\
\text { COMPETENZA }\end{array}$ & Geometria & \\
\hline Sapere e riconoscere & & \\
\hline Eseguire e applicare & & \\
\hline Esplorare e provare & $\begin{array}{l}\text { Stimare le dimensioni del parco della sede scolastica a partire da una sua ripresa } \\
\text { aerea, calcolando un rapporto di scala dell'immagine in base ad alcune misure } \\
\text { di lunghezza comunemente conosciute (automobili, pista 100 m, panchine ecc.) }\end{array}$ \\
\hline $\begin{array}{l}\text { Matematizzare } \\
\text { e modellizzare }\end{array}$ & \multicolumn{2}{|l|}{} \\
\hline $\begin{array}{l}\text { Interpretare e } \\
\text { riflettere sui risultati }\end{array}$ & $\begin{array}{l}\text { Riflettere sulla possibilità che la defor- } \\
\text { mazione prospettica influisca in manie- } \\
\text { ra significativa sulle misure stimate }\end{array}$ & $\begin{array}{l}\text { Verificare I'accettabilità dei risultati } \\
\text { in base alla normativa NAR }\end{array}$ \\
\hline $\begin{array}{l}\text { Comunicare } \\
\text { e argomentare }\end{array}$ & $\begin{array}{l}\text { Spiegare al resto della classe la strategia ideata dalla coppia per la stima } \\
\text { delle dimensioni del parco }\end{array}$ \\
\hline $\begin{array}{l}\text { Competenze } \\
\text { trasversali }\end{array}$ & $\begin{array}{l}\text { - Collaborazione } \\
- \text { Comunicazione } \\
- \text { Pensiero critico e creativo }\end{array}$ \\
\hline $\begin{array}{l}\text { Contesti di } \\
\text { formazione generali }\end{array}$ & $\begin{array}{l}\text { - Vivere assieme ed educazione alla cittadinanza } \\
- \text { Tecnologia e media }\end{array}$ \\
\hline
\end{tabular}

\subsection{Allenamento e sviluppo: definizione delle operazioni di lancio Obiettivo progettuale}

Definire la sequenza delle operazioni di lancio per garantire il corretto funzionamento di tutti i sistemi, la sicurezza di persone e cose e il successo della missione.

\section{Tempistiche e materiale}

Un'ora-lezione. Codice NAR; razzomodelli; rampa di lancio; starter; dispositivi di protezione individuali; materiale per il calcolo dell'apogeo.

\section{Descrizione dell'attività}

All'interno di ciascun gruppo gli allievi sono stati suddivisi secondo diversi ruoli che sarebbero stati ricoperti anche durante il lancio dei razzomodelli:

- Responsabile della sicurezza (prima e durante il lancio).

- Responsabile rampa di lancio (corretti assemblaggio e installazione del modello).

- Responsabile del motore a razzo (corretta installazione del motore e sua accensione).

- Responsabili meteo e rilevamento apogeo (verifica delle condizioni atmosferiche e misurazioni angolari).

Dopo aver preso coscienza del proprio ruolo, agli allievi è stato chiesto di stendere una check-list che descrivesse la sequenza logica di tutte le operazioni e i controlli da svolgere per il corretto lancio del razzomodello, indicando anche per ogni punto il responsabile che avrebbe dovuto svolgere/monitorare l'operazione. Per far ciò gli allievi avevano a disposizione tutti gli artefatti, i dispositivi e gli strumenti che avreb- 
bero poi potuto utilizzare il giorno del lancio.

Al termine di questa prima fase le diverse proposte dei gruppi sono state condivise con una messa in comune e si è confezionata una check-list finale condivisa da tutti i team (Allegato 8).

Inquadramento nel Piano di studio della scuola dell'obbligo ticinese

Questa attività non mobilita competenze specificatamente matematiche, ma mobilita quelle trasversali, operando inoltre in contesti di formazione generali che stimolano inoltre il pensiero logico e computazionale (Tabella 10).

Tabella 10

Allenamento e sviluppo:

definizione delle

operazioni di lancio.

Inquadramento nel Piano

di studio della scuola

dell'obbligo ticinese.

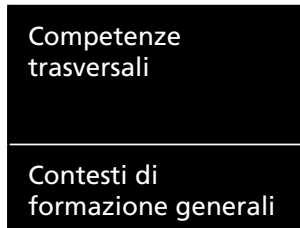

- Comunicazione

- Collaborazione

- Pensiero riflessivo e critico

- Vivere assieme ed educazione alla cittadinanza

\subsection{Realizzazione: installazione e lancio}

Obiettivo progettuale

Preparare il campo di volo, lanciare il razzomodello e determinarne la quota di apogeo.

\section{Tempistiche e materiale}

Due ore-lezione. Materiale per il lancio (nastro segnalatore, picchetti, rampa di lancio, razzomodelli, accenditori ecc.); per il calcolo dell'apogeo (pistole angolari, carta millimetrata, goniometri, righe, decametri, altimetro ecc.) e per la sicurezza (fischietto, bande "Remove before launch", occhiali di protezione ecc.).

\section{Descrizione dell'attività}

La prima ora-lezione è stata dedicata all'allestimento del campo di volo: gli allievi sono stati suddivisi nelle diverse tipologie di responsabili descritte nell'attività precedente e a ciascuno dei gruppi sono stati affidati compiti differenziati per la preparazione del campo di volo. In particolare:

- responsabili della sicurezza. Verifica della check-list, organizzazione delle zone di lavoro sul campo e verifica della bontà del lavoro degli altri gruppi in accordo alla check-list;

- responsabili della rampa di lancio. Installazione della rampa di lancio con la creazione di una recinzione regolare distante almeno 5 metri dalla rampa, utilizzando solo 4 asticelle e nastro bianco-rosso;

- responsabili meteo e rilevamento apogeo. Installazione e preparazione delle due stazioni di misura degli angoli, ripasso del metodo di determinazione dell'apogeo;

- responsabili motori. Addetti alle riprese fotografiche delle attività di preparazione, installazione del motore nel razzomodello e comando d'accensione al lancio. Alcune fasi dell'attività sono mostrate nella Figura 12, nella Figura 13 e nella Figura 14, mentre le schede operative si possono ritrovare nell'Allegato 9 . 


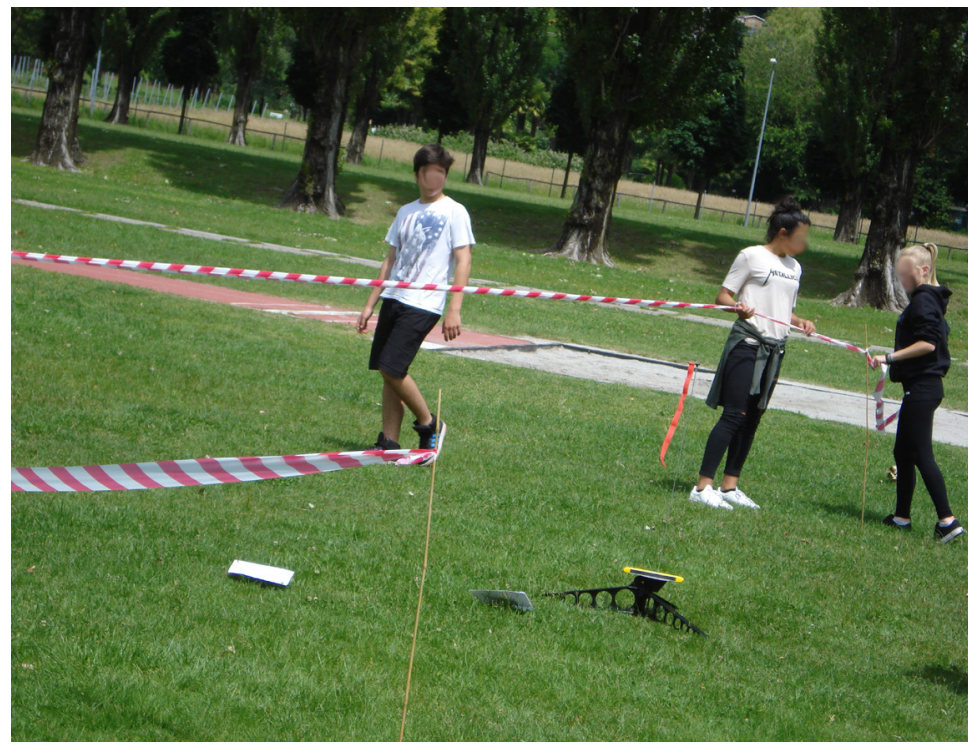

Figura 13

Preparazione del campo

di volo.
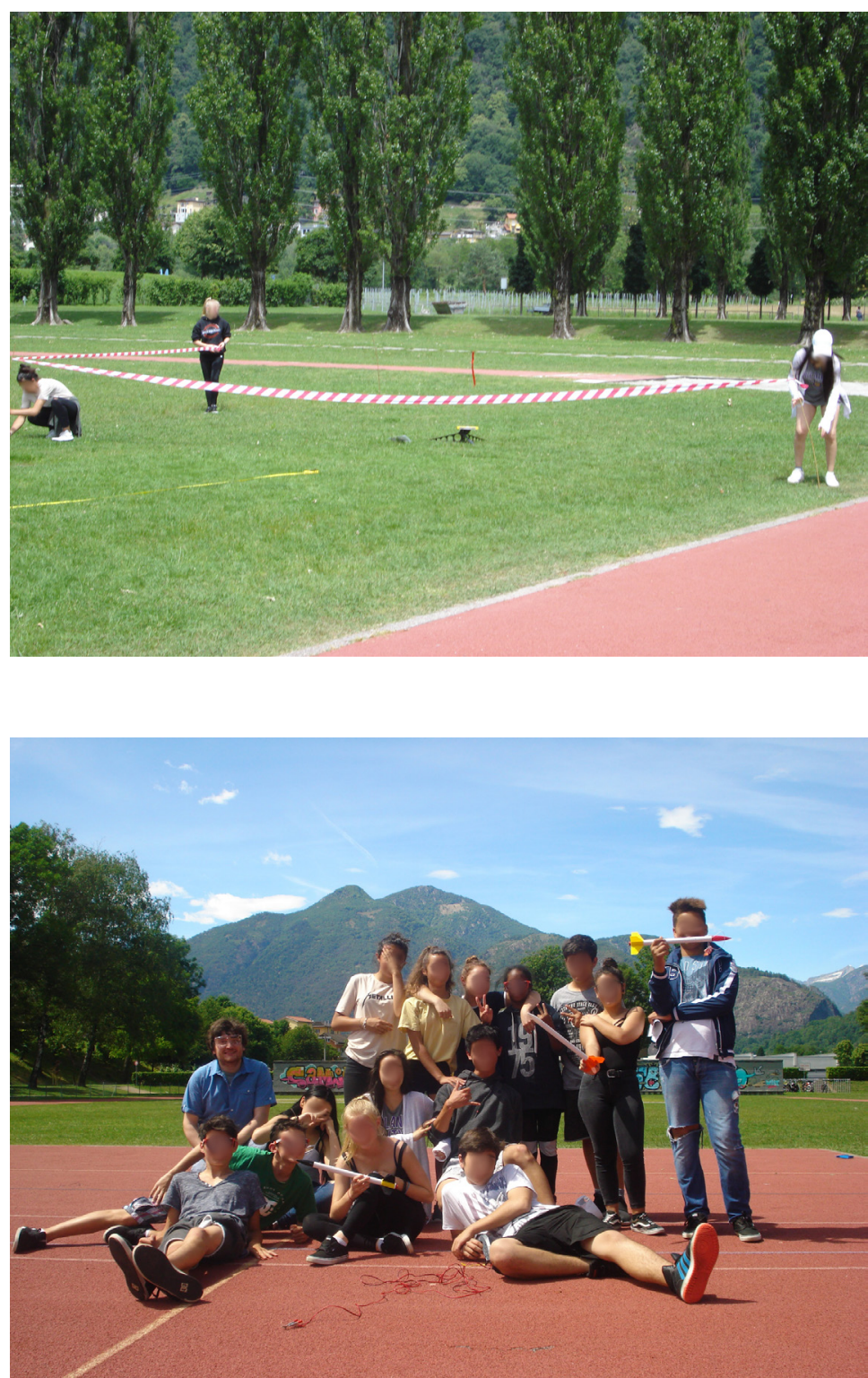

Foto di gruppo di classe con i razzomodelli. 
Nella seconda ora-lezione si sono svolti i lanci dei razzomodelli di tutti i gruppi (Figura 15), la raccolta delle misurazioni effettuate e la determinazione degli apogei raggiunti nei diversi voli. L'entusiasmo degli allievi nella preparazione e lancio dei loro razzomodelli, unitamente alla percezione di stare concretizzando un lavoro preparato per mesi, ha contributo a creare un clima di lavoro disteso, motivante e concentrato.

Figura 15

Un razzomodello pronto sulla rampa di lancio.

Tabella 11

Realizzazione:

installazione e lancio.

Inquadramento nel Piano

di studio della scuola

dell'obbligo ticinese.

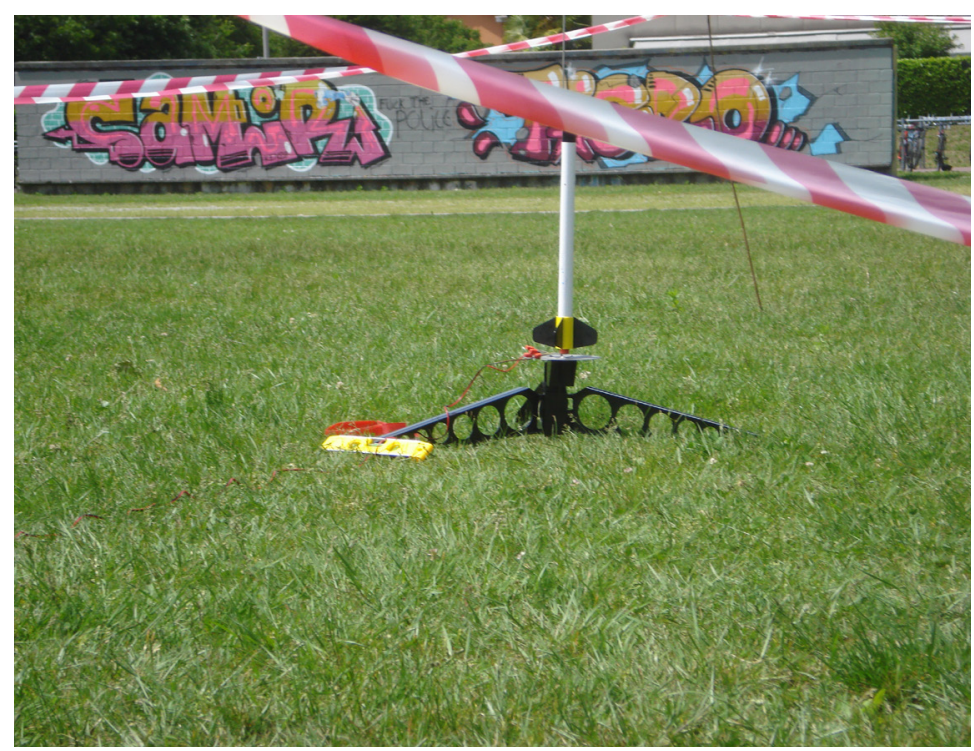

Inquadramento nel Piano di studio della scuola dell'obbligo ticinese

Questa attività - in particolare quella dei responsabili della rampa di lancio e delle stazioni di misura - si inserisce negli ambiti Geometria e Grandezze e misure. La cultura della sicurezza promossa durante questa attività si inserisce invece nel contesto di formazione generale di Vivere assieme ed educazione alla cittadinanza.

\begin{tabular}{|c|c|c|}
\hline $\begin{array}{l}\text { MODELLO DI } \\
\text { COMPETENZA }\end{array}$ & Geometria & Grandezze e misure \\
\hline \multicolumn{3}{|l|}{ Sapere e riconoscere } \\
\hline Eseguire e applicare & $\begin{array}{l}\text { Applicare le caratteristiche geometriche } \\
\text { del quadrato (in particolare la relazione } \\
\text { lato-diagonale) }\end{array}$ & $\begin{array}{l}\text { Misurare lunghezze utilizzando il } \\
\text { decametro }\end{array}$ \\
\hline Esplorare e provare & \multirow{2}{*}{\multicolumn{2}{|c|}{$\begin{array}{l}\text { Realizzare una recinzione quadrangolare regolare garantendo una distanza } \\
\text { minima del contorno dal centro di } 5 \text { metri }\end{array}$}} \\
\hline $\begin{array}{l}\text { Matematizzare } \\
\text { e modellizzare }\end{array}$ & & \\
\hline \multicolumn{3}{|l|}{$\begin{array}{l}\text { Interpretare e } \\
\text { riflettere sui risultati }\end{array}$} \\
\hline \multicolumn{3}{|l|}{$\begin{array}{l}\text { Comunicare } \\
\text { e argomentare }\end{array}$} \\
\hline $\begin{array}{l}\text { Competenze } \\
\text { trasversali }\end{array}$ & \multicolumn{2}{|l|}{$\begin{array}{l}\text { - Collaborazione } \\
\text { - Comunicazione } \\
\text { - Pensiero creativo }\end{array}$} \\
\hline $\begin{array}{l}\text { Contesti di } \\
\text { formazione generali }\end{array}$ & \multicolumn{2}{|c|}{$\begin{array}{l}\text { - Vivere assieme ed educazione alla cittadinanza } \\
\text { - Tecnologia e media }\end{array}$} \\
\hline
\end{tabular}




\subsection{Realizzazione: analisi post-lancio}

\section{Obiettivo progettuale}

Ad ogni volo, in ciascun razzomodello è stato installato un altimetro-accelerometro per modellismo (Figura 16) per registrare con una frequenza di $20 \mathrm{~Hz}$ le misure di quota (calcolata internamente allo strumento a partire da una quota di riferimento e misurando la pressione atmosferica. Risoluzione $\pm 30 \mathrm{~cm}$ ) e di accelerazione raggiunte. I dati, scaricabili via bluetooth su uno smartphone, potevano essere visualizzati in un grafico cartesiano direttamente attraverso l'app fornita con l'altimetro (Figura 17) ed esportati come foglio elettronico.

Figura 16

Altimetro utilizzato

durante i lanci dei

razzomodelli.
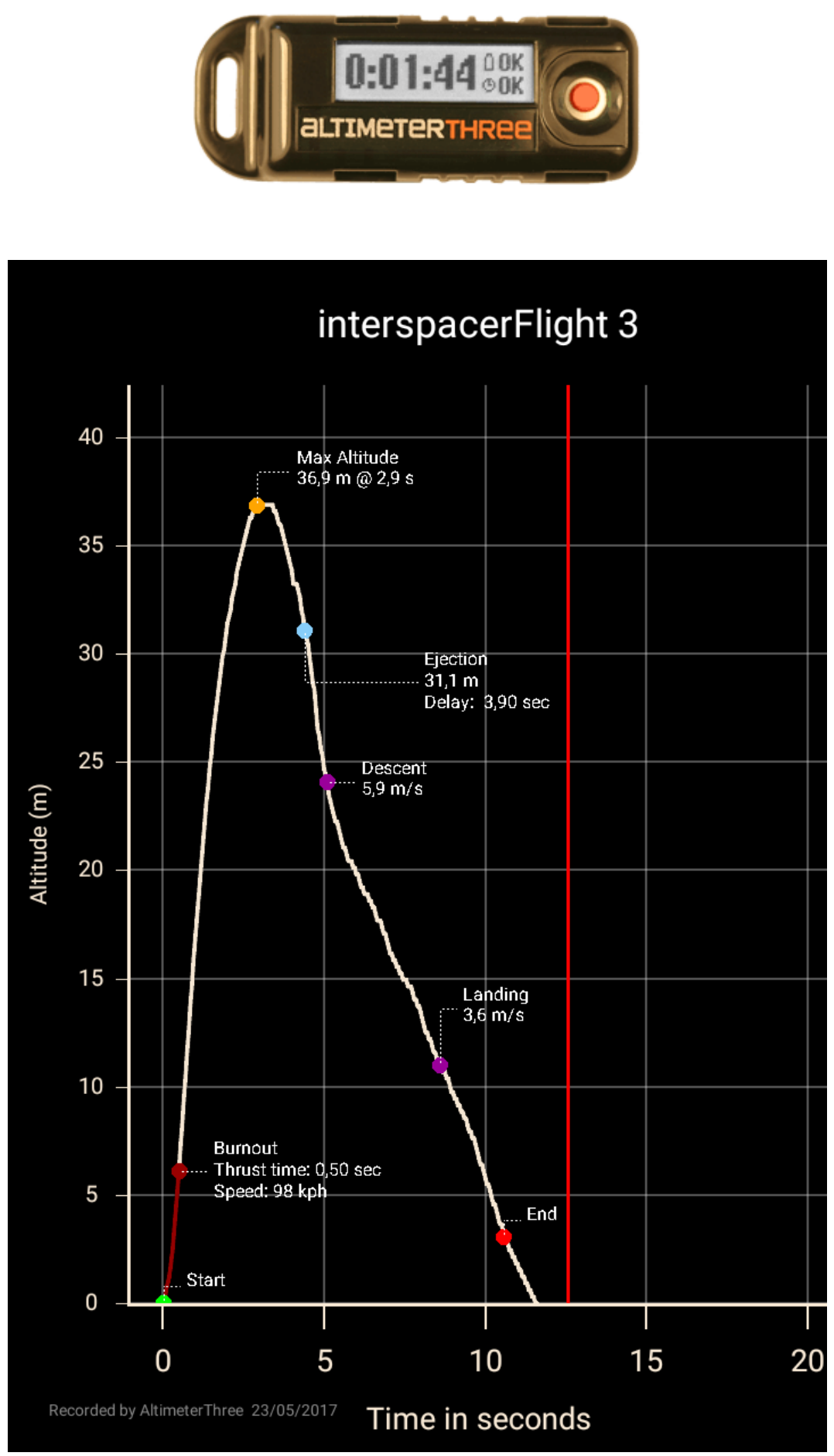
Gli obiettivi di questa fase sono riferiti al saper leggere i dati acquisiti dall'altimetro durante i voli, realizzare dei grafici a partire dal foglio elettronico e verificare l'errore percentuale compiuto durante la misurazione sul campo dagli allievi, utilizzando come riferimento la lettura fornita dall'altimetro.

\section{Tempistiche e materiale}

Due ore-lezione in aula di informatica. Altimetro; software Microsoft Exce/®; schede didattiche.

Due ore-lezione per la conclusione del film Cielo d'ottobre e discussione.

\section{Descrizione dell'attività}

Gli allievi sono stati suddivisi in coppie/terzetti di lavoro secondo I'appartenenza ai team di lancio. In aula di informatica il docente ha ripreso gli elementi fondamentali del piano cartesiano e ha fatto svolgere agli allievi semplici esercizi di attivazione. In seguito, ogni gruppo doveva determinare l'apogeo raggiunto dal proprio razzomodello secondo il metodo grafico già noto, in modo da verificare la bontà delle misurazioni compiute il giorno del lancio.

È stata poi lanciata la sfida di confrontare le misure ottenute con quelle effettuate dall'altimetro a bordo dei razzomodelli: il team che ha determinato la quota di apogeo con le pistole e il metodo grafico con maggior precisione - in termini di errore percentuale - rispetto ai dati dell'altimetro avrebbe vinto la sfida. Si è quindi reso necessario rappresentare graficamente i dati dello strumento, e questo è avvenuto grazie a un foglio elettronico Microsoft Exce/ß; l'attività è stata svolta da ogni coppia/terzetto di lavoro usando i dati relativi al proprio razzomodello e interpretando il grafico finale. Ai gruppi che terminavano velocemente il lavoro veniva chiesto di calcolare per ogni istante di tempo rilevato la velocità (noti la variazione di quota e di tempo) e di rappresentare graficamente i risultati per osservare l'andamento di questa grandezza durante il volo.

Si veda un esempio dei grafici ottenuti per un razzomodello nella Figura 18.

Figura 18

Grafico quota e velocità

di un razzomodello gene-

rato con foglio di calcolo

dai dati acquisiti con l'alti-

metro: altitudine (viola)

e velocità (azzurro) nel

tempo.

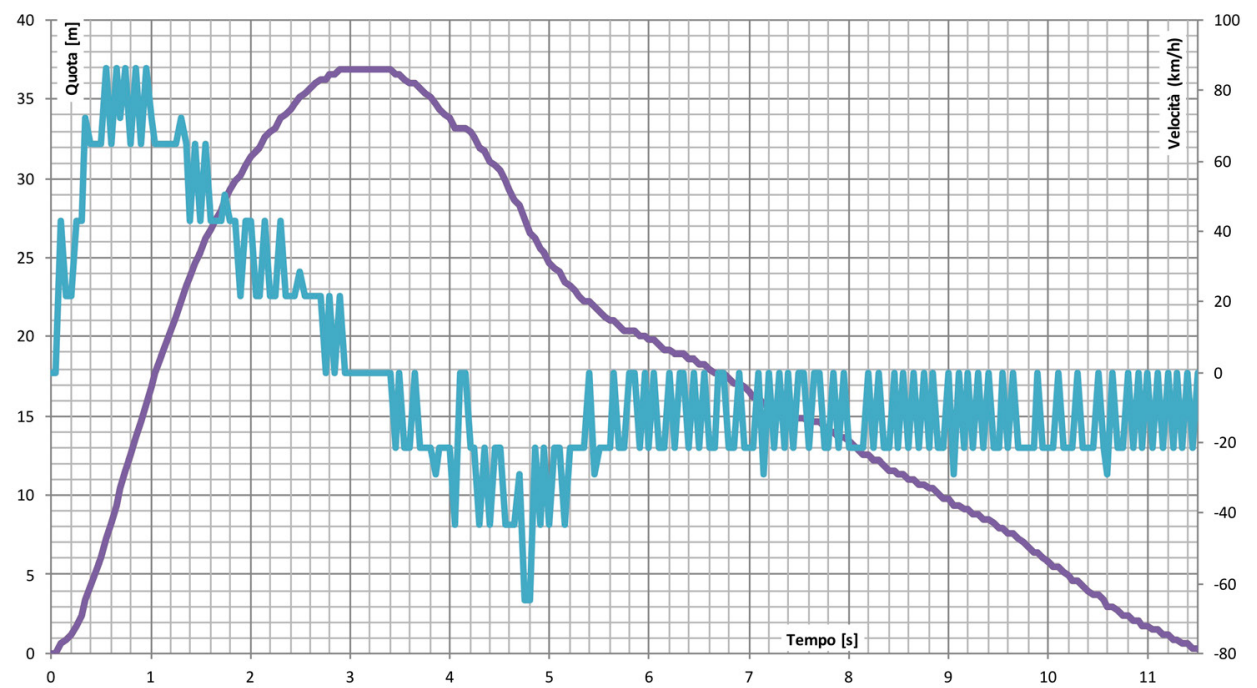

La scoperta della massima quota e della massima velocità raggiunta dal proprio razzomodello e la sfida del commettere il minor errore percentuale nel confronto fra le due misurazioni ha motivato molto l'intera classe, soprattutto confrontando i valori 
trovati con misure di distanza e velocità comuni all'esperienza quotidiana degli allievi. Le schede didattiche riferite a questa attività sono disponibili nell'Allegato 10. Al termine di tutto l'itinerario è stata conclusa in due ore-lezione la visione del film Cielo d'ottobre, lasciando uno spazio finale per risonanze, condivisioni e libero confronto sull'intero percorso svolto e sulle scelte di vita e i desideri professionali degli allievi: seppur non determinante, il percorso ha contribuito a una maggior presa di coscienza da parte degli allievi riguardo le loro scelte dopo la scuola dell'obbligo, soprattutto nella valutazione critica e non superficiale dei consigli ricevuti dagli adulti (genitori, docenti, amici,...).

Inquadramento nel Piano di studio della scuola dell'obbligo ticinese Questa attività si inserisce negli ambiti Numeri e calcolo, Grandezze e misure e Funzioni.

Tabella 12

Realizzazione: analisi post-lancio. Inquadramento nel Piano di studio della scuola dell'obbligo ticinese.

\begin{tabular}{|c|c|c|c|}
\hline $\begin{array}{l}\text { MODELLO DI } \\
\text { COMPETENZA }\end{array}$ & Numeri e calcolo & Grandezze e misure & Funzioni \\
\hline Sapere e riconoscere & & Definire la velocità & \\
\hline Eseguire e applicare & $\begin{array}{l}\text { Confrontare le percen- } \\
\text { tuali fra valori numerici }\end{array}$ & & $\begin{array}{l}\text { Leggere, completare e } \\
\text { utilizzare un diagramma } \\
\text { cartesiano }\end{array}$ \\
\hline \multicolumn{4}{|l|}{ Esplorare e provare } \\
\hline $\begin{array}{l}\text { Matematizzare } \\
\text { e modellizzare }\end{array}$ & & & $\begin{array}{l}\text { Sintetizzare in un } \\
\text { diagramma cartesiano } \\
\text { (tempo-spazio-velocità) } \\
\text { il profilo di missione del } \\
\text { razzomodello }\end{array}$ \\
\hline $\begin{array}{l}\text { Interpretare e } \\
\text { riflettere sui risultati }\end{array}$ & & \multicolumn{2}{|c|}{$\begin{array}{l}\text { Interpretare il diagramma cartesiano e confrontare } \\
\text { i valori delle grandezze in gioco (tempo, quota, } \\
\text { velocità) con misure prossime alla vita quotidiana } \\
\text { degli allievi }\end{array}$} \\
\hline $\begin{array}{l}\text { Comunicare } \\
\text { e argomentare }\end{array}$ & & & \\
\hline $\begin{array}{l}\text { Competenze } \\
\text { trasversali }\end{array}$ & \multicolumn{3}{|l|}{ - Collaborazione } \\
\hline $\begin{array}{l}\text { Contesti di } \\
\text { formazione generali }\end{array}$ & \multicolumn{3}{|c|}{$\begin{array}{l}\text { - Tecnologie e media } \\
\text { - Scelte e progetti personali }\end{array}$} \\
\hline
\end{tabular}

\section{Conclusioni, riflessioni critiche e possibili sviluppi}

Il progetto Razzi di classe ha permesso di perseguire in maniera soddisfacente il traguardo di competenza e quelli di apprendimento dell'allievo presentati nel primo paragrafo di questo articolo: gli allievi hanno manifestato entusiasmo e motivazione lungo I'intero itinerario, mostrando un modesto incremento dell'interesse anche in ore di lezione di matematica esterne al progetto. Le conoscenze, le abilità e le competenze matematiche mobilitate hanno visto un'applicazione reale e pratica, fortemente finalizzata a uno scopo preciso e accattivante come il lancio di un raz- 
zomodello. Circa gli aspetti di competenza più trasversali - la collaborazione e la disponibilità alla riflessione per la propria progettualità di vita - si è osservato un miglioramento parziale, soprattutto da parte di alcuni allievi, fermo restando che un itinerario di qualche ora-lezione può fornire soltanto una piccola e circoscritta occasione di attivazione e riflessione personali rispetto al quotidiano.

Il tempo dedicato al progetto in griglia oraria è risultato adeguato ed equilibrato rispetto alla programmazione annuale e ai traguardi di apprendimento per la classe terza.

La progettazione dell'intero itinerario si è fortemente appoggiata sul concetto di differenziazione, a diversi livelli:

- Dal punto di vista del docente. Poiché il progetto è stato entusiasmante fin dalla prima fase di ideazione, il docente ha potuto coinvolgere specificatamente la propria formazione, il proprio vissuto e la propria passione per la tematica, contribuendo positivamente alla progettazione di un itinerario che potesse essere accattivante per la classe.

- Dal punto di vista della disciplina. Le attività prettamente matematiche presenti nell'itinerario si sono strutturate con differenziazione progressiva e nell'ottica dell'apprendimento cooperativo (attività a coppie, a gruppi, a terzetti, jigsaw);

- Dal punto di vista degli allievi. La suddivisione continua di ruoli e compiti molto eterogenei fra loro in un'ottica cooperativa ha permesso di stimolare ciascun allievo secondo le proprie competenze disciplinari e trasversali, valorizzandone la presenza operativa all'interno di un contesto collaborativo di lavoro.

L'itinerario avrebbe potuto essere più valorizzato in termini di comunicazione esterna, sia all'interno della sede scolastica - al fine di raggiungere una maggior visibilità e gratificazione scolastica - sia all'interno delle famiglie - per stimolare una maggior presa di coscienza degli allievi dell'importanza e l'interesse del compito che stavano perseguendo. Oltre a ciò, la potenzialità offerta dall'efficace cornice narrativa del film Cielo d'ottobre è stata poco sfruttata nella riflessione personale con gli allievi. Tutto ciò si sarebbe potuto favorire anche implementando possibili ulteriori sviluppi del progetto Razzi di classe, fra i quali:

- progettazione di ulteriori attività matematiche connesse al progetto (eventualmente con traguardi di apprendimento ridotti o implementati per adattare l'itinerario a classi diverse dalla terza base). A tal proposito si vedano Milligan (1999; 2000) e Nolte (2012);

- una maggiore interdisciplinarità e quindi il coinvolgimento attivo di più docenti (scienze, arti plastiche, italiano, storia, educazione alla cittadinanza, geografia);

- lo sviluppo più strutturato di un percorso di conoscenza di sé e di educazione alle scelte da svolgere in altre ore didattiche o in ora di classe, sfruttando la cornice narrativa e di senso fornita dalla visione del film Cielo d'ottobre.

L'auspicio è di poter revisionare e approfondire eventuali implementazioni ed espansioni del progetto Razzi di classe.

Il progetto non ha inciso radicalmente sulla motivazione e sul profitto degli allievi nelle ore di matematica, né tanto meno ha contribuito in maniera decisiva e centrale allo sviluppo di sé dei singoli. Ad ogni modo ha certamente contribuito disciplinarmente e trasversalmente nel suo piccolo a fare alzare agli allievi un poco lo sguardo al cielo della loro vita, fornendo un apporto che si è andato ad aggiungere all'intera esperienza scolastica e al vissuto personale.

Ma d'altronde già i latini affermavano... per aspera ad astra! 


\section{Bibliografia}

Barrowman, J. (1970). Stability of a model rocket in flight. Centuri. Phoenix (Arizona): Centuri Engineering Company.

Comoglio, M., \& Cardoso, M. A. (1996). Insegnare e apprendere in gruppo. /l Cooperative Learning. Roma: Libreria Ateneo Salesiano.

DECS. (2015). Piano di studio della scuola dell'obbligo ticinese. Tratto da ScuolaLab: Disponibile in http://www.pianodistudio.ch (consultato il 28.04.2019).

Gentile, M. (1998). Motivare ad apprendere. ISRE, 5(2), 80-109.

Grimm, A. (1999). Model Rocketry Study Guide. Estes Educator. Launch Learning Fun. Centuri Corporation.

Hickam, H. (1999). Cielo d'ottobre. Milano: Rizzoli.

Licheri, L.M. (2019, febbraio 20). La pedagogia del progetto. Metodo per lo sviluppo di competenze del XXI secolo e la diffusione dei valori europei. Disponibile in http://com munity.eseceducation.eu/c/document library/get file? groupld $=20182 \&$ folderld $=44462 \&$ title=Output+Teachers+O1+Italian+version.pdf (consultato il 28.04.2019).

Milligan, T. V. (1999). 69 science fair projects with model rockets. Colorado Springs (CO): Apogee Components Inc.

Milligan, T. V. (2000). Model rocket design and construction. How to create and build unique and exciting model rockets that work! Colorado Springs (CO): Apogee Components Inc.

Nolte, S. (2012). Mathematics and Model Rockets. A Teacher's Guide and Curriculum for Grades 5-12. Estes Educator. Launch Learning Fun. Estes Education.

Sepúlveda, L. (2010). Storia di una gabbianella e del gatto che le insegnò a volare. Milano: Salani.

Stine, H. G. (2004). Handbook of model rocketry. Hoboken (NJ): John Wiley \& Sons Inc.

\section{Autore/Diego Santimone}

Scuola media di Losone - Svizzera

diego.santimone@edu.ti.ch

\footnotetext{
Il razzimodellismo può essere pericoloso! Alcune norme di sicurezza (non esaustive) da rispettare sempre:

1) utilizzare materiali ed equipaggiamenti certificati e prodotti da ditte di modellismo professionale;

2) verificare la bontà della fattura del razzo modello e il soddisfacimento delle posizioni di CG e CP;

3) rispettare rigorosamente il regolamento NAR;

4) rispettare le misure di sicurezza più elementari (distanze, dispositivi di protezione individuali, protezioni ecc.);

5) fare qualche prova in bianco senza gli allievi per conoscere il funzionamento ed il comportamento del razzomodello.

In caso di dubbi affidatevi a ditte di modellismo specializzate ed esperti del settore.

Non si improvvisa, mai!
} 